\title{
New Magnetics Observations with a Random Exchange Interaction
}

\author{
Alioune Aidara Diouf*, Souleymane Sene, Bassirou Lo \\ Physics Department, Faculty of Sciences and Techniques, University Cheikh Anta Diop of Dakar, Dakar-Fann Dakar, Senegal \\ Email address: \\ aliouneaidara.diouf@ucad.edu.sn(A. A. Diouf) \\ ${ }^{*}$ Corresponding author \\ To cite this article: \\ Alioune Aidara Diouf, Souleymane Sene, Bassirou Lo. New Magnetics Observations with a Random Exchange Interaction. American \\ Journal of Electromagnetics and Applications. Vol. 7, No. 2, 2019, pp. 19-24. doi: 10.11648/j.ajea.20190702.12
}

Received: November 19, 2019; Accepted: December 5, 2019; Published: December 11, 2019

\begin{abstract}
The aim of this paper is to investigate a new type of magnetic material, which has a permanent random exchange interaction. The ferromagnetic properties (magnetizations) of an Ising nanostructure are investigated using the effective field theory with correlations. The system has consisted from spin-1/2 atoms with a random exchange interaction $J_{i j}$. The value of $J_{i j}$ is randomly distributed by a random function. A specific investigation about the special effects of the random core exchange interaction on the magnetization and the critical temperature has been studied. For the appropriate value of the system parameter new descriptions and phenomena of the magnetizations in 3D have been obtained. The results show that it is possible to get the same ferromagnetic behavior observed with a constant exchange interaction by using a permanent random exchange interaction. Moreover, the results found can be as well displayed in three dimensions (3D) with the same behavior observed in $2 \mathrm{D}$. The results are well detailed in the paper.
\end{abstract}

Keywords: Random Exchange Interaction, Effective Field Theory, Magnetization in 3D

\section{Introduction}

During the last years, magnetic nanoparticles systems [1-6] have attracted considerable attention of researchers due to their several applications in the new technologies [7-14]. Indeed, when the dimension of the magnetic nanoparticles decreases to the nanometer scale, the surface to volume ratio increases. Because of this process, these nanoparticles start to exhibit new phenomenon and interesting physical properties which are totally different from those observed in the bulk materials [15]. Some numerous experimental techniques have been reported to get nanoscales magnetic materials [16-19] and theoretically, the magnetic properties of these nanoparticles have mainly been examined by using the mean-field theory (MFA), the effective-field theory (EFT), and the Monte Carlo simulation (MC) [20-29]. Otherwise, the exchange interaction is among the most important parameters in the magnetic properties, several authors have already worked on it with different forms $[30,15,22,26]$, moreover, almost all the scientific investigations in the magnetic field are done with a constant exchange interaction. On the other hand, just a few groups of scientists used the influence of the random exchange interaction to investigate some magnetic properties such as M. F. Thrope and al. which investigated the phase diagram of a ferromagnetic system by using a random exchange interaction [31, 32]. Experimentally, M. Drillon and al. investigated the magnetic behavior of "rigid" ferrimagnetic chains and model their results by using the influence of the random exchange interaction in the ferrimagnetic Heisenberg Chain [33]. The aim in this paper is to investigate the ferromagnetic properties (magnetizations) by the EFT within the probability distribution technique that accounts for the self-spin correlations by using the random exchange interaction. On the other hand, as far as one knows, the magnetic properties of the Ising nanosystems obtained in this paper, have not yet been described in the literature with a random exchange interaction. In this paper, one presents a new method to observe the ferromagnetic magnetizations in three dimensions.

\section{Model and Formalism}

Consider the nanostructure defined by the following structure. 


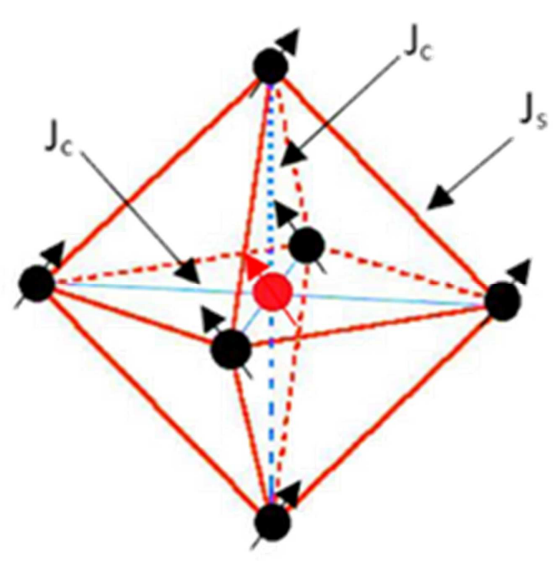

Figure 1. Nano-octahedral structure.

The red atom is the central spin and the black atoms play the role of the nearest neighbors. In this system, the exchanges interactions $\left(\mathrm{J}_{\mathrm{s}}\right)$, which linked the atoms on the surface are neglected. Consider only the internal exchange interaction $\mathrm{J}_{\mathrm{c}}=\mathrm{J}_{\mathrm{ij}}$ as a random exchange interaction. Otherwise, the method used to establish the ordered moment is the EFT, well detailed in [34]. Therefore, the transverse Ising model used to investigate the ferromagnetism properties is defined by:

$$
H=-\sum_{(i j)} J_{i j} S_{j z} S_{i z}-\Omega \sum_{i} S_{i x}-h \sum_{i} S_{i z}
$$

where $H=-A S_{i z}+B S_{i x}$

$$
A=\sum_{(i j)} J_{i j} S_{j z}+h
$$

and

$$
B=-\Omega \text {. }
$$

The random exchange interaction is defined by $J_{i j}=$ rand (x) with rand () a random function, $x$ the number of exchange interaction possibility, $S_{i z}$ and $S_{i x}$ the components following $(\mathrm{Oz})$ and $(\mathrm{Ox})$ of the spin $\mathrm{S}=1 / 2, h$ the magnetic field and $\Omega$ the transversal field. Within the formulation of the EFT, accordingly, the different steps for the calculations of the magnetic properties of the system are well detailed in [34, 35].

$S_{i z}$ and $S_{i x}$ are represented by the following matrix:

$$
S_{i z}=\left(\begin{array}{cc}
\frac{1}{2} & 0 \\
0 & -\frac{1}{2}
\end{array}\right) \text { and } S_{i x}=\left(\begin{array}{cc}
0 & \frac{1}{2} \\
\frac{1}{2} & 0
\end{array}\right)
$$

To calculate the different averages, one has to find the eigenvalues and the associated vectors of the Hamiltonian $\mathrm{H}$ [34], which is represented by the following matrix:

$$
H=\left(\begin{array}{cc}
\frac{1}{2} & \frac{1}{2} \\
\frac{1}{2} & -\frac{1}{2}
\end{array}\right)
$$

and the corresponding vectors are given by:

$$
\begin{gathered}
E_{1} \longrightarrow\left|\Psi_{1}\right\rangle=a_{1}\left|\frac{1}{2}\right\rangle+b_{1}\left|\frac{1}{2}\right\rangle \\
E_{2} \longrightarrow\left|\Psi_{2}\right\rangle=a_{2}\left|\frac{1}{2}\right\rangle+b_{2}\left|-\frac{1}{2}\right\rangle
\end{gathered}
$$

the quantities $\left|\frac{1}{2}\right\rangle$ and $\left|-\frac{1}{2}\right\rangle$ are the associated vectors of $\mathrm{S}_{\mathrm{z}}$. The coefficients $a_{k}, b_{k}(k=1,2)$ can be calculated from the following equations [34]:

$$
H\left|\Psi_{k}\right\rangle=E_{k}\left|\Psi_{k}\right\rangle
$$

and one has

$$
\left(\begin{array}{cc}
\frac{A}{2} & \frac{B}{2} \\
\frac{B}{2} & -\frac{A}{2}
\end{array}\right)\left(\begin{array}{l}
a_{k} \\
b_{k}
\end{array}\right)=\left(\begin{array}{l}
a_{k} \\
b_{k}
\end{array}\right)\left|\Psi_{k}\right\rangle
$$

Taking account of the conditions of the following normalization of the states:

$$
\left\langle\Psi_{k} \mid \Psi_{k}\right\rangle=a_{k}^{2}+b_{k}^{2}=1
$$

Thus, one has:

$$
a_{k}^{2}=\frac{B^{2}}{\left(A-2 E_{k}\right)^{2}+B^{2}} b_{k}^{2}=\frac{\left(A-2 E_{k}\right)^{2}}{\left(A-2 E_{k}\right)^{2}+B^{2}}
$$

From statistics of spin systems, for the operators $S_{i r}^{\alpha}$ at site i, one has:

$$
\left\langle S_{i r}^{\alpha}\right\rangle=\left\langle\frac{\operatorname{Tr}\left(S_{i r}^{\alpha} \exp (-\beta H)\right.}{\operatorname{Tr}(\exp (-\beta H)}\right\rangle
$$

Where the angular bracket $\langle\ldots\rangle$ denotes a canonical thermal average, $\beta=1 / \mathrm{k}_{\mathrm{B}}$. T, T is the temperature and $\alpha$ is the number of order moment. If the exchange interactions are restricted to nearest-neighbors interactions only, the evaluation of the inner traces over selected spins in the last equations yields is

$$
\left\langle S_{i r}^{\alpha}\right\rangle=\left\langle F_{\alpha}(A, B)\right\rangle
$$

Where 


$$
\left\langle F_{\alpha}(A, B)\right\rangle=\frac{A}{\sqrt{A^{2}+B^{2}}} \tanh \left(\frac{1}{2} \beta \sqrt{A^{2}+B^{2}}\right)
$$

if $r=z$ : Longitudinal and

$$
\left\langle F_{\alpha}(A, B)\right\rangle=\frac{B}{\sqrt{A^{2}+B^{2}}} \tanh \left(\frac{1}{2} \beta \sqrt{A^{2}+B^{2}}\right)
$$

if $r=x$ : Transversal

The above thermal averages are valid for a fixed spatial configuration. The step is to carry out the configurational average noted $\langle\ldots\rangle_{r}$, the quantities are the polarization $M^{\alpha}$ defined by:

$$
M^{\alpha}=\left\langle\left\langle S_{i r}^{\alpha}\right\rangle\right\rangle=\left\langle\left\langle F_{\alpha}(A, B)\right\rangle\right\rangle
$$

$M^{\alpha} \quad$ is a function depending of $\sum_{i=0}^{N} S_{i r}$ like: $F\left(\sum_{i=0}^{N} S_{i r}\right)$. One introduces the Dirac delta function:

$$
\begin{aligned}
& \delta\left(y_{1}-\sum_{i=0}^{N} S_{i r}\right)=\int \frac{d \lambda_{1}}{2 \pi} e^{i\left[y_{1}-\sum_{i=0}^{N} S_{i r}\right]} \\
& \delta\left(y_{1}-\sum_{i=0}^{N} S_{i r}\right)=\int \frac{d \lambda_{1}}{2 \pi} e^{-i \lambda_{1} S_{i r}}
\end{aligned}
$$

One has:

$$
\begin{gathered}
\left\langle F\left(\sum_{i=0}^{N} S_{i r}\right)\right\rangle=\int d y_{1}\left(y_{1}-\sum_{i=0}^{N} S_{i r}\right) F\left(y_{1}\right) \\
F\left(\sum_{i=0}^{N} S_{i r}\right)=\int d y_{1} F\left(y_{1}\right) \int \frac{d \lambda_{1}}{2 \pi} \prod_{i=1}^{N} e^{-i \lambda_{1} S_{i r}}
\end{gathered}
$$

In the approximation of Zernike decoupling of the multiple correlations, one has:

$$
\left\langle F\left(\sum_{i=0}^{N} S_{i r}\right)\right\rangle=\int d y_{1} F\left(y_{1}\right) \int \frac{d \lambda_{1}}{2 \pi} \prod_{i=1}^{N}\left\langle e^{-i \lambda_{1} S_{i r}}\right\rangle
$$

To perform the configurational average on the right-hand side of the last equation, one uses the probability distribution method based on the use of generalized Van der Wander identities [38]. Once done we have:

$$
\prod_{i=1}^{N}\left\langle e^{-i \lambda_{1} S_{i r}}\right\rangle=\prod_{i=1}^{N}\left(\sum_{S_{i r}=-1 / 2}^{1 / 2} P\left(S_{i r}\right) e^{-i \lambda_{1} S_{i r}}\right)
$$

Where the distribution functions $V\left(S_{i r}\right)$ are given by:

$$
V\left(S_{i r}\right)=\frac{1}{2}\left[\left(1-2 p_{z}\right) \delta_{S_{i r,-1 / 2}}+\left(1+2 p_{z}\right) \delta_{S_{i r, 1 / 2}}\right]
$$

Taking into account relations above, one gets the following relations for the order moments:

$$
\begin{aligned}
& M^{\alpha}=\left\langle\left\langle F_{\alpha}(A, B)\right\rangle\right\rangle=2^{-(N+1)} \sum_{\mu_{1}=0}^{N} C_{\mu_{1}}^{N}\left(1+p_{z}\right)^{N-\mu_{1}} \\
& \left(1-p_{z}\right)^{\mu_{1}} \operatorname{th}\left(J_{\alpha}\left(\left(N-2 * \mu_{1}\right)+h\right)\right)
\end{aligned}
$$

In this equation, $\mathrm{N}$ represents the coordination number and $C_{k}^{n}$ is the binomial coefficients, $C_{k}^{n}=\frac{n !}{k !(n-k) !}$ one has the self-consistent equations for the order moments $M^{\alpha}$ with the system, which can be solved directly by numerical iteration without any further algebraic manipulations. Then the average total polarization is given by:

$$
M=\frac{\sum_{i=1}^{n} M_{i}^{z}}{N}
$$

With $\mathrm{N}$ the number of spin in the structure represented in the Figure 1.

\section{Results and Discussions}

In certain magnetic materials, the magnetism is obtained by the coupling of the magnetic moments and form magnetically ordered states. The coupling is the exchange interaction which in nature quantum mechanical and is rooted in the overlap of electrons in conjunction with Pauli's exclusion principle.

In the present paper one has used only a direct internal random exchange operates "rand ()" between moments, which are close for having sufficient overlap of theirs waves functions. The coupling provides a short range and strong coupling, which decrease rapidly and randomly as the ions are separated. A simple way to understand a direct random exchange interaction is to look at two atoms with one electron each. Otherwise, when the interatomic distance is small, the electrons spend most of their time in between neighboring atoms. In the case where the atoms are far apart, the electrons spend their time away from each other in order to minimize the electron-electron repulsion.

In the following paper, one has used a nanosystem where we consider only the internal exchange interaction $\left(\mathrm{J}_{\mathrm{C}}\right)$ with a null surface exchange interaction $\left(\mathrm{J}_{\mathrm{S}}=0\right)$. As you know, the phase transition from ferromagnetic to paramagnetic depends on the temperature and particularly from the Curie Temperature Tc. Otherwise, each ferromagnetic system has its own Curie temperature $(\mathrm{Tc})$. Knowing that fact one investigated by comparison two cases, a case where the exchange interaction $J i j$ is constant and another case where $J i j$ is random by using our system describes by a spin $S=1 / 2$, a transverse field $\Omega / J=1.00$, an external field $h / J=0.0$. The aim of the comparison was to investigate the behaviors of the magnetization according to the temperatures with two different types of exchange interaction as depicted in figure 2 . 


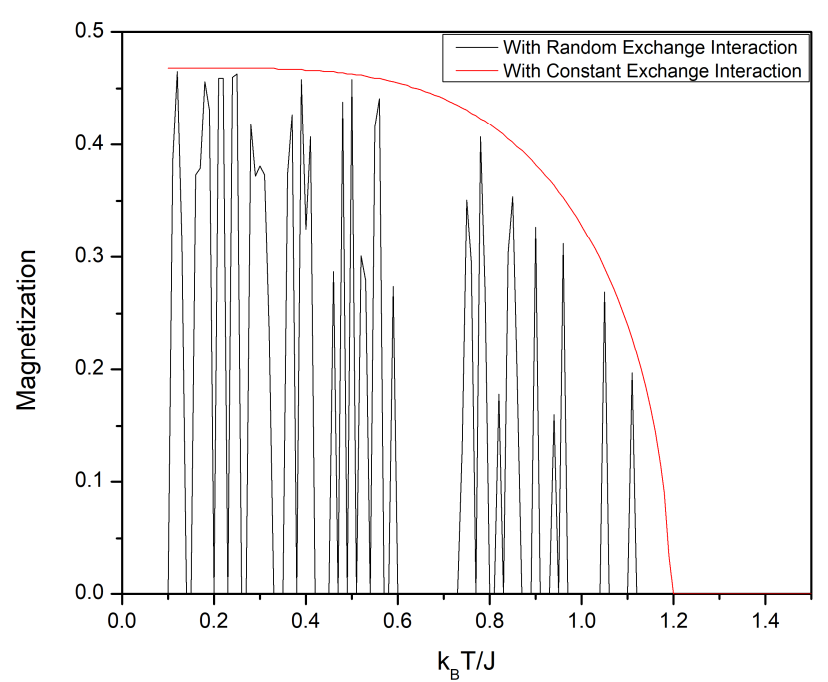

Figure 2. Thermal variation of the magnetization with random and constant exchange interaction.

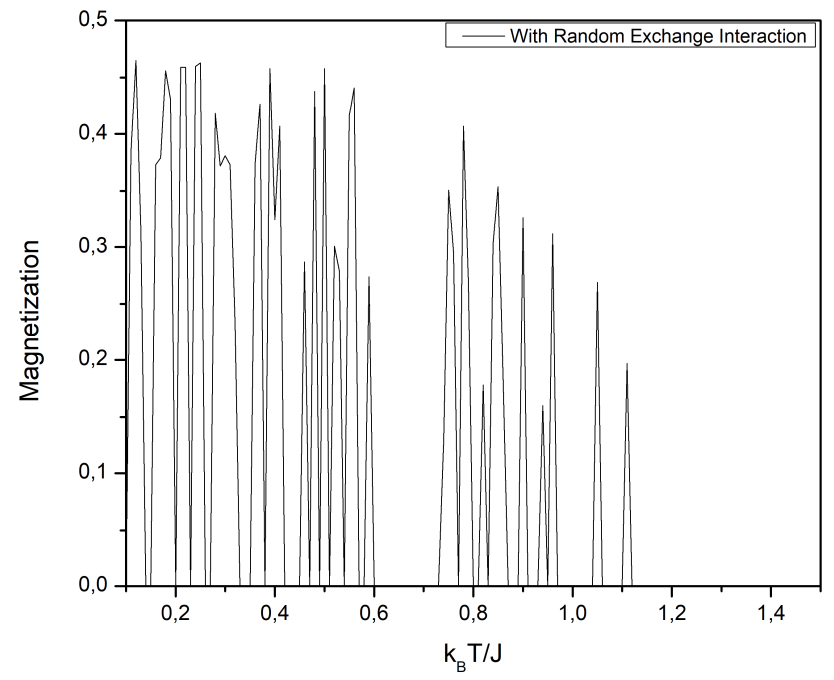

Figure 3. Thermal variation of the magnetization with a random exchange interaction for $x=2$.

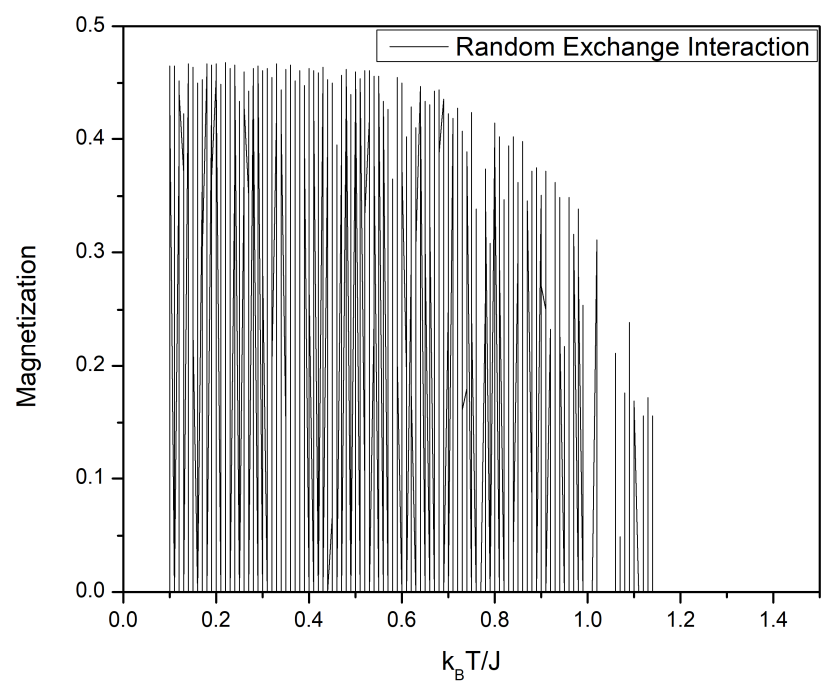

Figure 4. Thermal variation of the magnetization with a random exchange interaction for $x=10$.

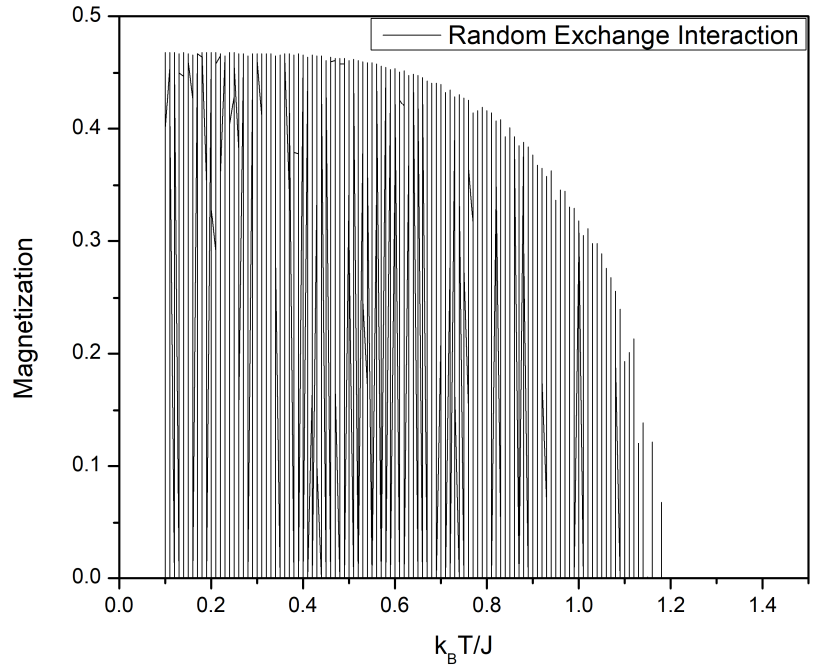

Figure 5. Thermal variation of the magnetization with a random exchange interaction for $x=100$.

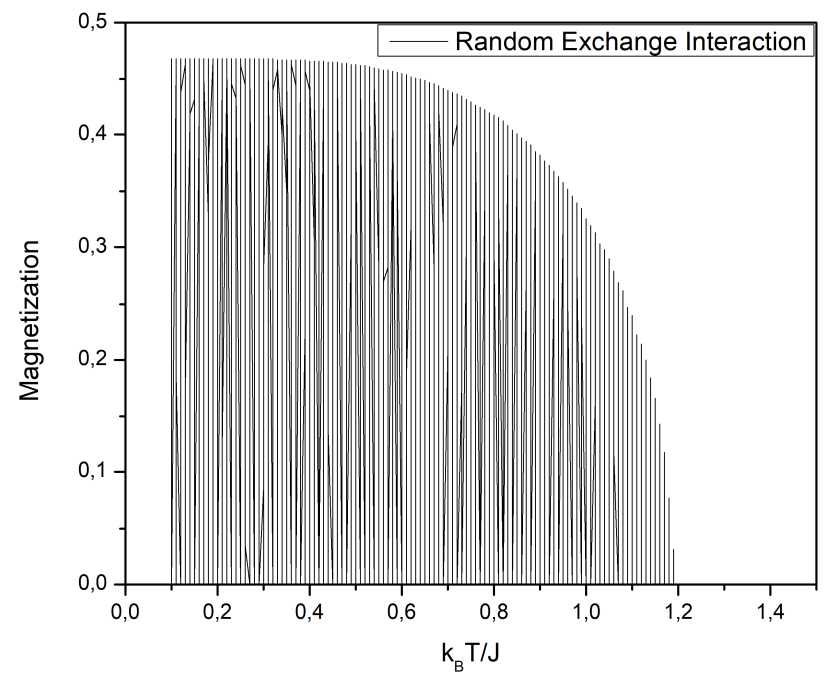

Figure 6. Thermal variation of the magnetization with a random exchange interaction for $x=1000$.

For describing the behavior of the magnetization in three dimensions (3D), one has used several exchange interaction possibilities $x=2,10,100,1000$ for the random function Jij=rand $(x)$. One has demonstrated that more the random possibility is important more the signature of the magnetization is well displayed as shown in figures $3,4,5$ and 6.

The ferromagnetic materials are sensitive to the temperatures, above the Curie temperature $\left(T_{C}\right)$ the materials lose its ferromagnetism properties and become merely paramagnetic. As with the paramagnetic particles, the ferromagnetic atoms have a permanent magnetic moment but are strongly oriented to the crystallographic axes, unlike the paramagnetic particles. One can explain this phenomenon by the fact, that in a single crystal it exists domains where all the magnetic moments are parallel. And they are linked by exchange interaction, taken randomly in the present paper and are aligned with a particular axis. Otherwise, from a domain to another all the moments are parallel to each other, 
but they may be aligned with a different axis. Thus, one has a number of domains, each highly magnetized, with some domains magnetized in one direction and some in another.

The random exchange interaction used is the origin of the internal field magnetic field, which reflects the electrostatic Coulomb repulsion of the electrons on neighboring atoms and the Pauli principle. According to the spin configuration, it exists an energy difference between them. That is why the random effects of the exchange interaction and the influence of the temperature cause a decrease in the spontaneous magnetization at higher temperatures, which cause as well the increasing excitation of spin waves with a shape of the magnetizations curves. The fluctuation of the spin from ferromagnetism to paramagnetic is random that is why we observed the unusual magnetization curves displayed in the Figures 2, 3, 4, 5 \& 6 .

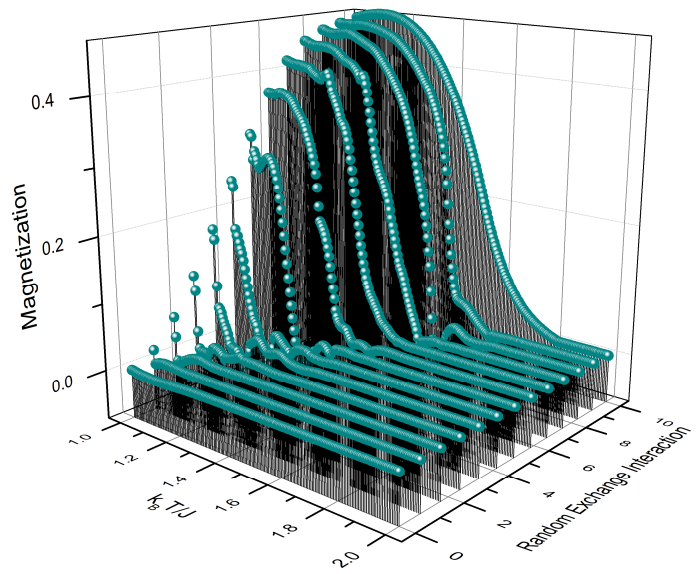

Figure 7. Thermal variation of the magnetization in $3 D$ with a random exchange interaction for $x=1$.

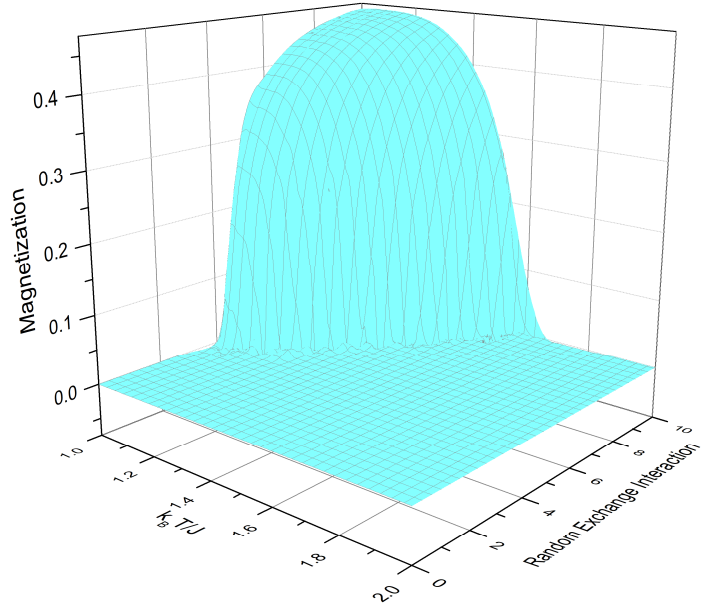

Figure 8. Thermal variation of the magnetization in $3 D$ with a random exchange interaction for $x=10$.

Otherwise, according to the temperature $\mathrm{kBT} / J$, the transverse field $\Omega / J$ and the random exchange interaction $J i$ used, one has computed and represented the results of the magnetization as a matrix function $F(x, y, z)$. Where $x$ is the temperature, $y$ is the random exchange interaction and $z$ the results of the magnetization. For plotting the three dimensions (3D) magnetization, the matrix $F(x, y, z)$ has been diagonalized and the results are displayed in figures 7 , $8,9 \& 10$.

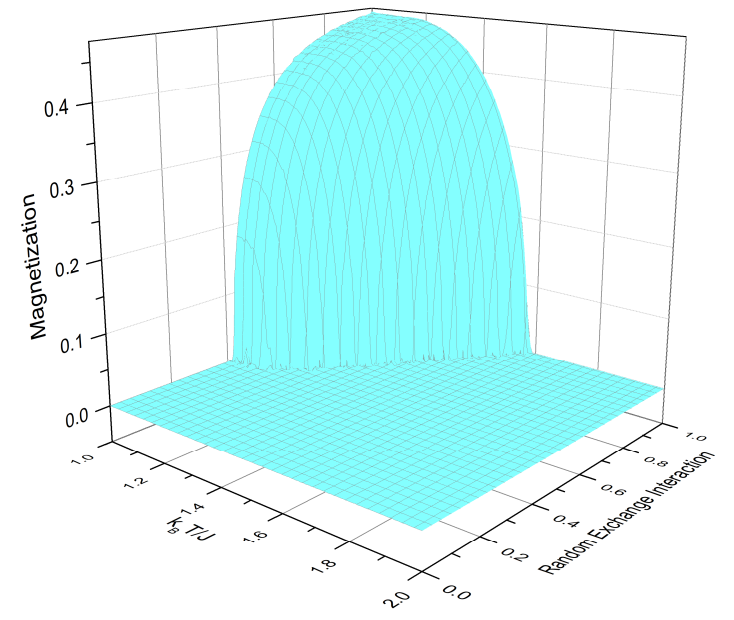

Figure 9. Thermal variation of the magnetization in $3 D$ with a random exchange interaction for $x=100$.

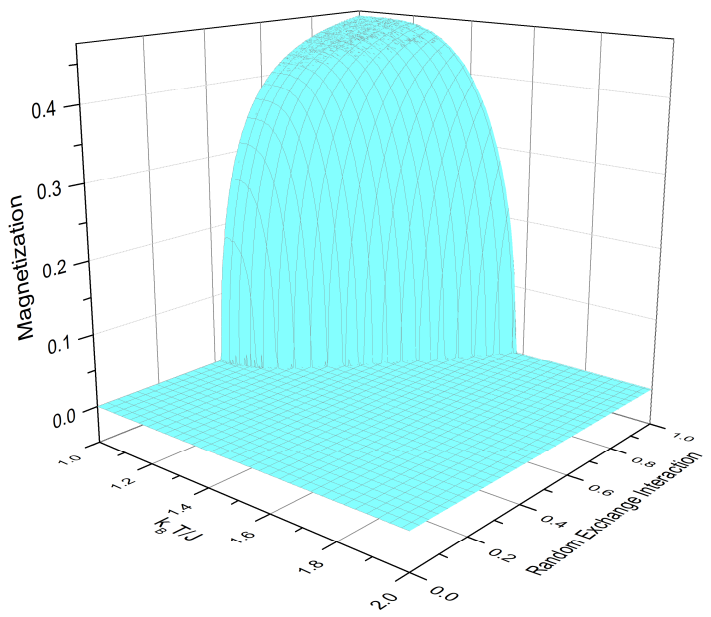

Figure 10. Thermal variation of the magnetization in $3 D$ with a random exchange interaction for $x=1000$.

\section{Conclusion}

In the present paper, we've investigated an octahedral nano-system. With one spin at the center and six spins on the surface connected via a random internal exchange interaction. The surface exchange interaction is neglected. The effects of the random internal exchange interaction have been explained. New ferromagnetic behavior have been observed and are well detailed. We've shown that with a random exchange interaction and another ferromagnetic behavior can be obtained. Otherwise, the model, Theory, and steps used for the results in 3D are well detailed in the article, in reference to the authors [34]. The study of the new phenomena observed with a random internal exchange interaction open a new field in the experimental research of magnetism. 


\section{Acknowledgements}

I thank Pr. Aboubaker Chedikh Beye and his lab for their help about my researches at the Cheikh Anta Diop University of Dakar.

\section{References}

[1] E. Tirosh and G. Markovich, Adv. Mater. 19 (2007) 2608.

[2] R. Q. Song, A. W. Xu, and S. H. Yu, J. Am. Chem. Soc. 129 (2007) 4152 .

[3] H. Gao, M. Staruch, M. Jain, P. X. Gao, P. Shimpi, Y. Guo, W. Cai, H. J. Lin, Appl. Phys. Lett. 98 (2011) 123105.

[4] [4] Alioune Aidara Diouf, Bassirou Lo, Alhadj Hisseine Issaka Ali, Aboubaker Chedikh Beye. Comparative Investigation and Generalized of the Core/Shell Effects on the Magnetics Properties in the Ferromagnetic Cubic Nanoparticles by the Transverse Ising Model. American Journal of Nanomaterials. Vol. 4, No. 1, 2016, pp. 1-7. http://pubs.sciepub.com/ajn/4/1/1.

[5] M. J. Benitez, O. Petracic, E. L. Salabas, F. Radu, H. Tüysüz, Schüth, and H. Zabel, Phys. Rev. Lett. 101 (2008) 097206. X. Qi, W. Zhong, Y. Deng, C. Au, Y. Du, CARBON 48 (2010) 365 .

[6] H. Zeng, J. Li, J. P. Liu, Z. L. Wang, S. Sun, Nature 420 (2002) 395 .

[7] A. Lopez-Ortega, M. Estrader, G. Salazar-Alvarez, A. G. Roca and J. Nogues, Physics Reports (2014), http://dx.doi.org/10.1016/j.physrep.2014.09.007.

[8] R. H. Kodama, J. Magn. Magn. Mater. 200 (1999) 359.

[9] G. V. Kurlyandskaya, M. L. Sanchez, B. Hernando, V. M. Prida, P. Gorria, M. Tejedor, Appl. Phys. Lett. 82 (2003) 3053.

[10] S. D. Bader, Reviews of Modern Physics 78 (2006) 1.

[11] J. Kim, H. S. Kim, N. Lee, T. Kim, H. Kim, T. Yu, I. C. Song.

[12] K. Moon, T. Hyeon, Angew. Chem. 47 (2008) 8438.

[13] A. H. Habib, C. L. Ondeck, P. Chaudhary, M. R. Bockstaller, and M. E. McHenry, J. Appl. Phys. 103 (2008) 07A307.

[14] M. I. Shukoor, F. Natalio, M. N. Tahir, V. Ksenofontov, H. A. Therese, P. Theato, H. C. Schröder, W. E. G. Müller, W. Tremel, Chem. Commun. 44 (2007) 4677.

[15] M. El Hamri, S. Bouhou, I. Essaoudi, A. Ainane, R. Ahuja, Investigation of the surface shell effects on the magnetic properties of a transverse antiferromagnetic Isingnanocube, Superlattices and Microstructures (2015), doi: http://dx.doi.org/10.1016/j.spmi.2015.01.003.

[16] J. Liu, Q. Li, T. Wang, D. Yu, and Y. Li, Angew. Chem. 116 (2004) 5158.

[17] S. Singhal, J. Singh, S. K. Barthwal, K. Chandra, J. Sol. Stat. Chem. 178 (2005) 3183.

[18] X. He, G. Song, J. Zhu, Mater. Lett. 59 (2005) 1941.

[19] K. Maaz, W. Khalid, A. Mumtaz, S. K. Hasanain, J. Liu, J. L. Duan, Physica E 41 (2009) 593.

[20] Kantar, E.: Eur. Phys. J. B. 90, 152 (2017).

[21] Kaneyoshi, T.: Phase Tran. 86, 404 (2013).

[22] Zaim, A., Kerouad, M., Boughrara, M.: Solid St. Commun. 158,76 (2013).

[23] Dakir, O., El Kenz, A., Benyoussef, A.: Physica A 426, 45 (2015).

[24] Yalcin, O., Erdem, R., Ozum, S., Demir, Z.: J. Magn. Magn. Mater. 389, 120 (2015).

[25] Lu, Z. X.: Phase Tran. 89, 273 (2016).

[26] El Hamri, M., Bouhou, S., Essoudi, I., Ainame, A., Ahuja, R., Dujardin, F.: J. Phys.: Conf. Ser. 758, 012023 (2016).

[27] Vatansever, E., Yuksel, Y.: J. Magn. Magn. Mater. 441, 548 (2017).

[28] Kaneyoshi, T.: Phys. Stat. Sol. b 242, 2938 (2005).

[29] Wang, W., Chen, D. D., Lv, D., Liu, J. P., Peng, Z.: J. Phys. Chem. Solids 108, 39 (2017).

[30] Kaneyoshi, T. J Supercond Nov Magn (2018), https://doi.org/10.1007/s10948-018-4709-5.

[31] M. F. Thorpe, D. Beeman, Physical Review B, 14, 1 (1976).

[32] M. F. Thorpe. J. Phys. 36, 1177 (1975).

[33] M. Drillon, E. Coronado, D. Beltran, R Georges, J. Appl. Phys. 57, 3353 (1985); doi: 10.1063/1.335094.

[34] Jozef Strecka, Michal Jascur, A brief account of the Ising andIsing-like models: Mean-field, effective-field and exact results, Acta Physics Slovaca 65 (2015) 235-367.

[35] I. Essaoudi, K. Bärner, A. Ainane, M. Saber, Physica A 385 (2007) 208-22. 